\title{
REMOTE TEMPERATURE AND HUMIDITY MONITORING SYSTEM USING WIRELESS SENSOR NETWORKS
}

\author{
Varsha jaladi ${ }^{1}$, Guthula Ganga Raja Sekhar ${ }^{2}$, K.Raghava Rao $^{3}$ \\ ${ }^{I}$ BTech Student, dept. of Electronics and Computers, $K$ L University, Guntur, AP \\ ${ }^{2}$ BTech Student, dept. of Electronics and Computers, $K$ L University, Guntur, AP \\ ${ }^{3}$ Professor, dept. of Electronics and Computers, $K$ L University, Guntur, AP
}

\begin{abstract}
Today's world has become very advanced with smart appliances and devices like laptops, tablets, televisions. smart phones with different features and their usage has been enormously increasing in our day-to-day life. The technology advancement in Digital Electronics and Micro Electro Mechanical Systems. In this scenario the most important role is played by Wireless Sensor Networks and its development and usage in heterogeneous fields and several contexts. the home automation field and process control systems and health control systems widely uses wireless sensor networks. Moreover with WSN we can monitor environments and its conditions also. We are designing a protocol to monitor the environmental temperature and humidity at different conditions. The architecture is simple to construct and ease to implement and also has an advantage of low power consumption. The aim of our paper to describe and show how to create a simple protocol for environment monitoring using a wireless development kit. we are using advanced technology of crossbow motes and NESC Language Programming.
\end{abstract}

Keywords: Motes, WSN, sensor, TinyOS, Nesc.

\section{INTRODUCTION}

From few years, usage of sensors has been increasing in our everyday lives and fields like industrial automation and households. The advancement and development of low power semiconductor technologies. with these technology, it is possible to build up complex systems which runs with just tens of microwatts of power along with this the low power technology requires very little maintenance in battery replacement for the devices. This type of mechanism not only enhances the sensor network reliability but also reduces man the terms of cost and hours spent by man in maintaining the network. we are using standard protocol of IEEE 802.15.4 as it supports for very low-rate wireless personal area networks. We are going to design a WSN which consists of nodes and small motes which are programmable and also they are embedded with different and distinguished devices as they can be used to the parameters and establish a communication between the Motes. The main task of mote is to communicate between the gateway and motes. In this process the detected data is sent to the gateway which processes the information received. The WSN'S architecture and design need to meet some important requirements and characteristics:

Less energy consumption: In order to prolong the lifecycle of each node we to reduce the energy consumption.

Fault tolerance: Whenever there is an error in the system or an malfunction happens it have to react quickly. $\square$

Scalability: The system's capacity to manage the network topology dynamically.
The quality of service: Security, latency, Integrity of data and many more.

Self configuration: It is important to the network to detect its operations and should reduce any external operation on the network.

The standard protocol of IEEE 802.15.4 provides the layers of physical and MAC. The function of physical layer is to deal with data reception and transmission services. The physical layer deals with transmission and data reception services, and the interface management using radio utilizes the Clear Channel Assessment module (CCA),Link Quality Indication Module(LQI) and Energy Detection Energy Detection module (ED).The Industrial Scientific and Medical(ISM) is the frequency bandwidth used to communicate. The important layer is MAC which provides several functions as it helps in managing and creating the Personal Area Network which defines a frame of control and also to ensure channel access using CSMA. The Security algorithms, systems for acknowledgement and error correction mechanism. The topology of networks can be divided in to following ways:

- Tree: The hierarchical structure of sensor nodes represent the tree topology;

- Mesh: every node can communicates with each node directly ;

- Star: The central node is directly connected to each node directly. 
The main advantage of WSNs is its Flexibility which is used in many applications like industrial control process, environmental applications and traffic monitoring are some. The contents of the paper are organized as follows: In Section II The wireless Platform and technologies are described. In the next section the architecture and environment for implementation of WSN is displayed. The next section shows an example which we have implemented on an application. Finally, the last section summarizes the paper and proposes some future works that can be implemented.

\section{WIRELESS TECHNOLOGY AND PLATFORM}

The MIB520 Mote is used as a hardware platform and a MICAZ mote embedded on it. The MICAZ mote is consisted of Microcontroller with ATMEL MEGA128L Low Power Microcontroller. The Wireless protocol complaint we use is IEEE 802.15.4 Which is an excellent choice as it helps in implementing the network with low cost and low power consumption. The MICAZ Mote comes with 26-pin connector inorder to interface with external hardware and sensor boards. The software we used is Moteworks which has further sub-parts of motecconfig, moteview, programming notepad. We can also use TinyOS version 2.0, which is an open source system. We have used Nesc programming for programming the motes.

The MICAZ mote manufacturer is by crossbow technologies which provide open source software of moteworks. The memsic technologies provide the data regarding the motes, sensor boards and its applications.

The moteworks software is designed to be user-friendly as its main aim is to provide a wireless network that supports Crossbow selection of motes and sensors of wireless platforms. The advantage of using TinyOS,

Moteworks is that they are easily understood and ease to work with Wireless Communication Mesh platform and architecture is easy to implement. The very advanced, dependable and robust mesh network can be implemented with the general understanding and brief knowledge to an extent regarding the workings of hardware and communication protocols network.

\section{General Architecture of Mote:}

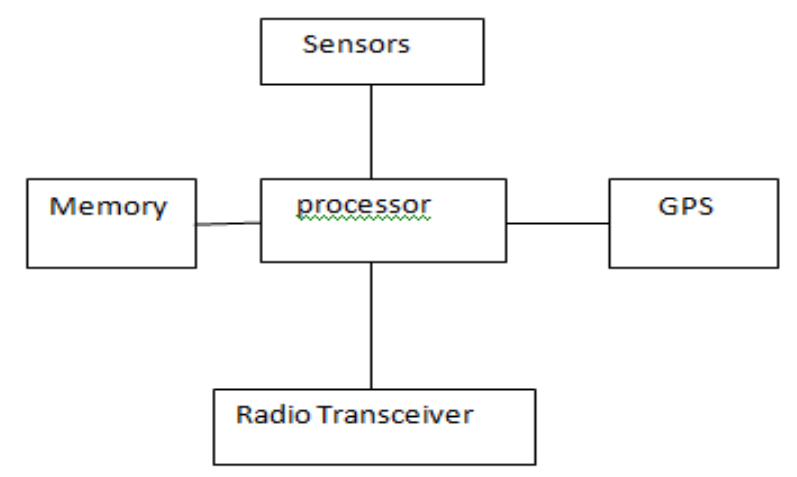

\section{DEVELOPMENT ENVIRONMENT}

The protocol is designed in a way in order to create a architecture in the form of network and routing strategy which is provided by application layer. The management of network and the commands should be executed with less power consumption at higher layer. The driver composes the is composed by the driver application layer of ZIGBEE platform and the code is stored into the ROM memory. The advanced generation sensor node can be operated and used with different operating system like TinyOS, conitiki, NanoRK. The conitiki operating system is multitasking which is used for embedded systems and older architectures. conitiki operating system is developed using $\mathrm{C}$ language for microcontrollers. The other operating system Nano-RK is real-time OS which works with sensor nodes consisting of particular hardware. The other operating system TinyOS is a operating system available in the open source and can be used in many sensor nodes. The TinyOS is flexible and totally event-based and is totally non-blocking and consists of many libraries. The language used Is NesC and the libraries all are connected to the source code directly.

\subsection{NESC Introduction}

The TinyOS system, libraries, and applications are written in nesC, a new language for programming structured component-based applications. The nesC language is primarily intended for embedded systems such as sensor networks. nesC has a C-like syntax, but supports the TinyOS concurrency model, as well as mechanisms for structuring, naming, and linking together software components into robust network embedded systems. The principal goal is to allow application designers to build components that can be easily composed into complete, concurrent systems, and yet perform extensive checking at compile time.

TinyOS defines a number of important concepts that are expressed in nesC. First, nesC applications are built out of components with well-defined, bidirectional interfaces. Second, nesC defines a concurrency model, based on tasks and hardware event handlers, and detects data races at compile time.

\section{Component Description:}

In particular hardware components used are:

- MICAZ mote: The MICAZ is a $2.4 \mathrm{GHz}$ mote module used for enabling low-power wireless sensor networks

- Base Station MIB520:A base station allows the aggregation of sensor network data on to the PC or the computer platform. Any Micaz Mote can function as a base station when it is connected to a standard PC interface or gateway board.

- MDA300[13]: The MDA300 Is a Data Acquisition Board. It consists of onboard humidity and temperature sensors. The proto-typing area supports connection to all 51pins on the expansion connector. 
In order to implement the WSN, we used Mote Works [15], a platform which provides an interface to simplify creation and monitoring of a WSN. Main software used are MoteConfig [16] and MoteView [17]. The first one provides a simple GUI to program Motes while the second one consists of an intuitive interface to create and manage the network.

\section{Architecture of Wireless Platform}

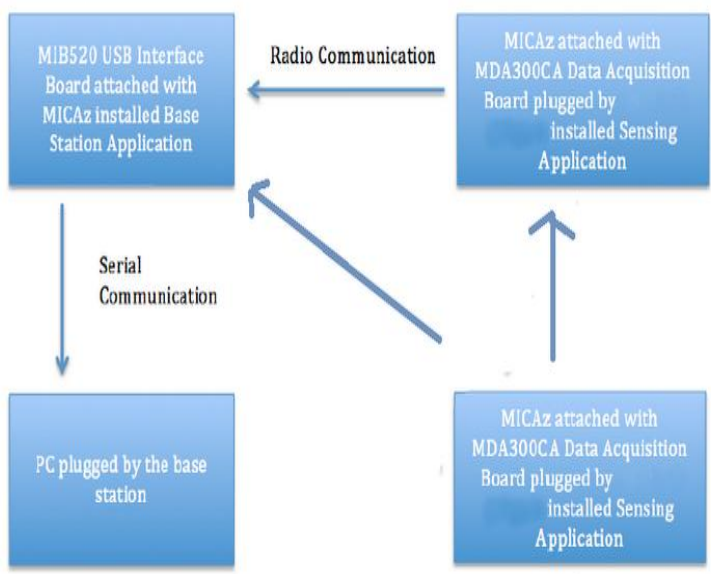

Initially, we need to program each mote with specified programs. The software Mote works provides a two way platform firmware to program the motes. we have to program the Gateway and Simple nodes with different programs depending on their functions. The central node which acts as a gateway is programmed to implement functions like:

- To gather and collect the data coming from different nodes;

- The data which is receives is sent to the base station and displays it on the monitor in which mote view is running.

Apart from gateway the other nodes are configured to provide the multihop routing and sensing data. we have used Moteconfig firmware for programming the nodes, represented in the Fig 1.

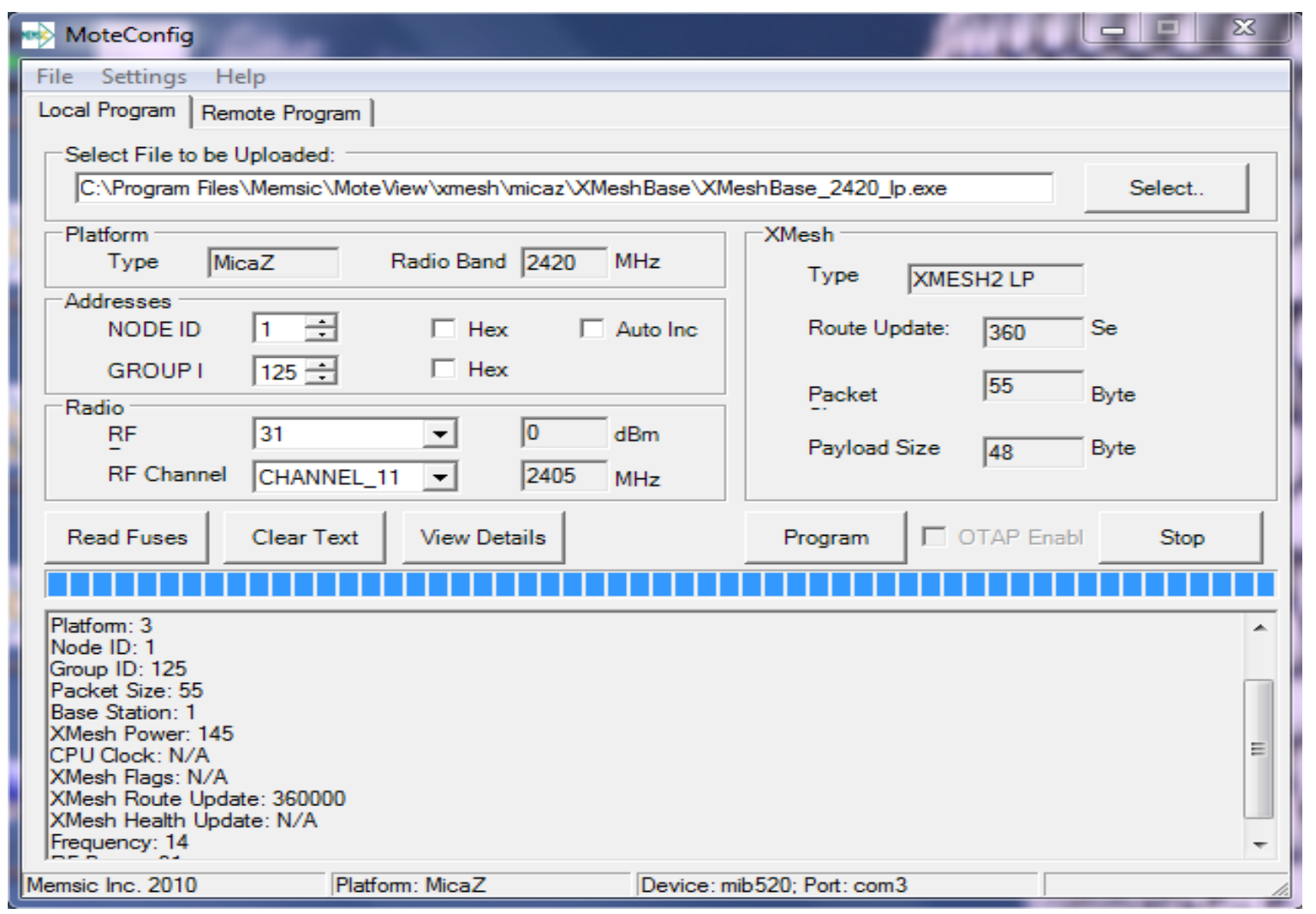

Fig. 1. MoteConfig GUI

After programming the motes we establish a connection of WSN using Moteview and data which is collected from all the nodes is displayed and information is stored in the Postgre $\mathrm{SQL}$ database. Moteview is a platform where we can monitor the gathered information as shown in the below Fig2. Another feature of moteview is it helps in multiple of view of data in the form of graphs, histogram and network topologies. 


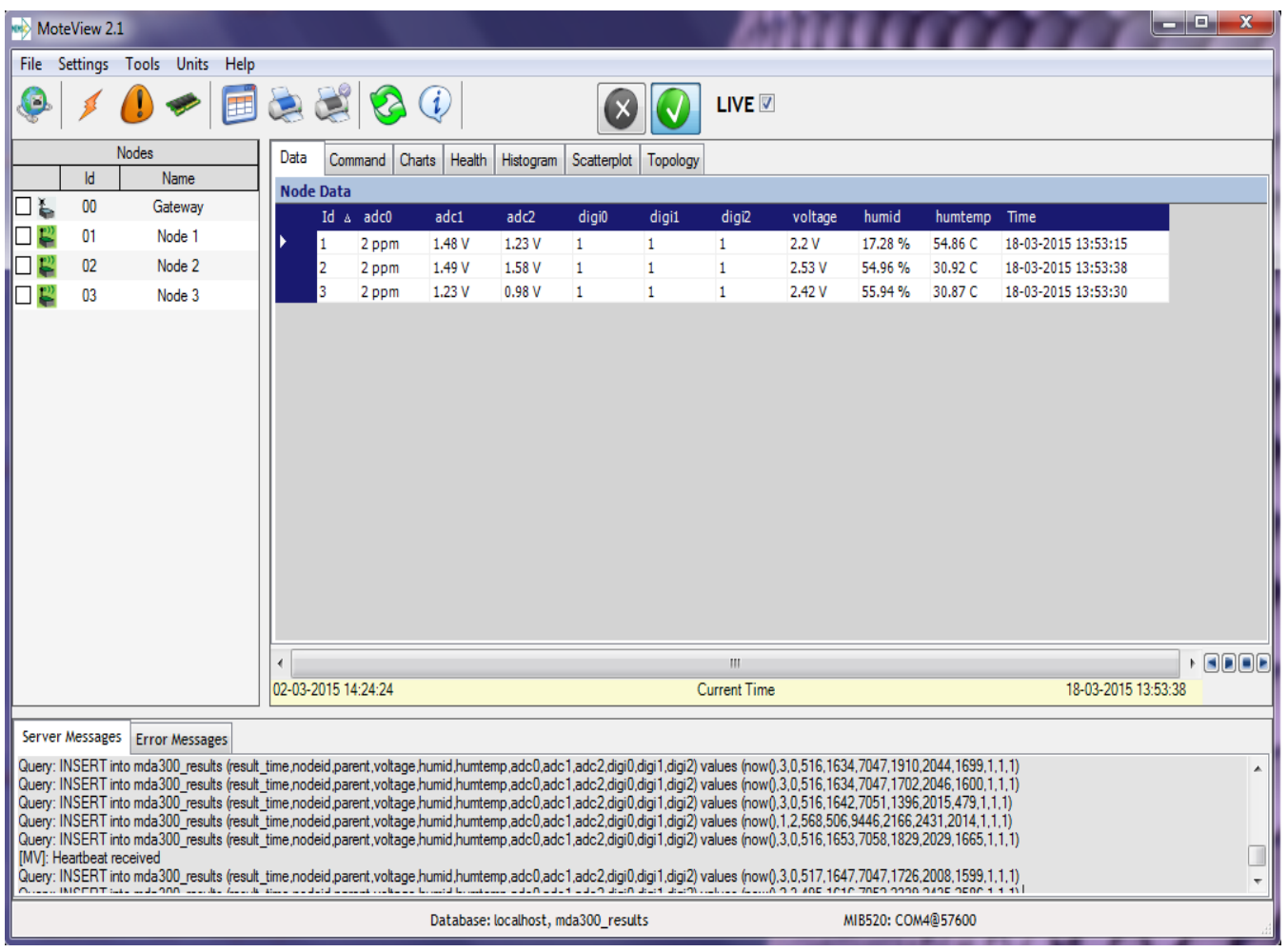

Fig. 2. MoteView Screenshot

\section{CASE STUDY}

Our network is constructed by using 3 motes having sensor boards (sensor nodes) and a base node (gate way) connected to computer. The Sensor nodes communicate with each other and the radio messages which are detected are sent to base station which is connected to the PC. One of the important feature of nodes is multi hopping which allows radio communication to extend a long range in order top cover wide area network. Now even when the nodes are not in the range the data messages can be delivered from one node to another node and these nodes forward the data to the gateway. Our paper implements the WSN to monitor temperature and humidity.
The MDA300 consists of onboard humidity and temperature sensors. The MDA300 needs $2.4 \mathrm{vdc}$ power supply and it consumes less power. In an active stage, the device utilizes only average current .The data acquisition board is attached to MICAZ mote and program is installed in it. The data obtained is stored into the results table .

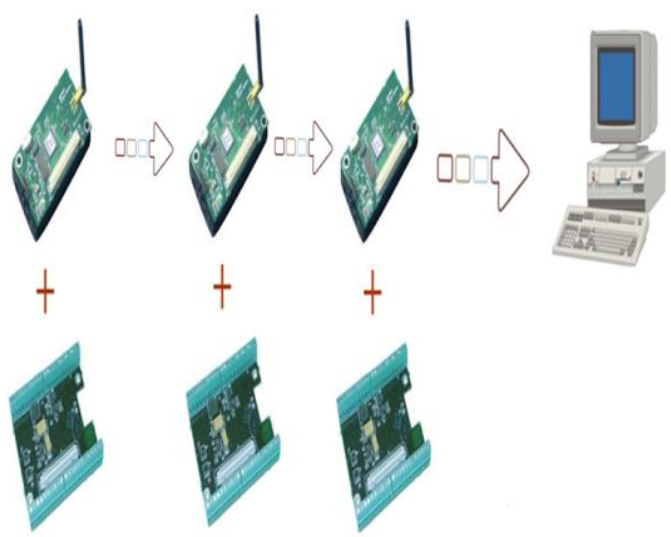

Fig.3. Design of Wireless sensor network with motes 


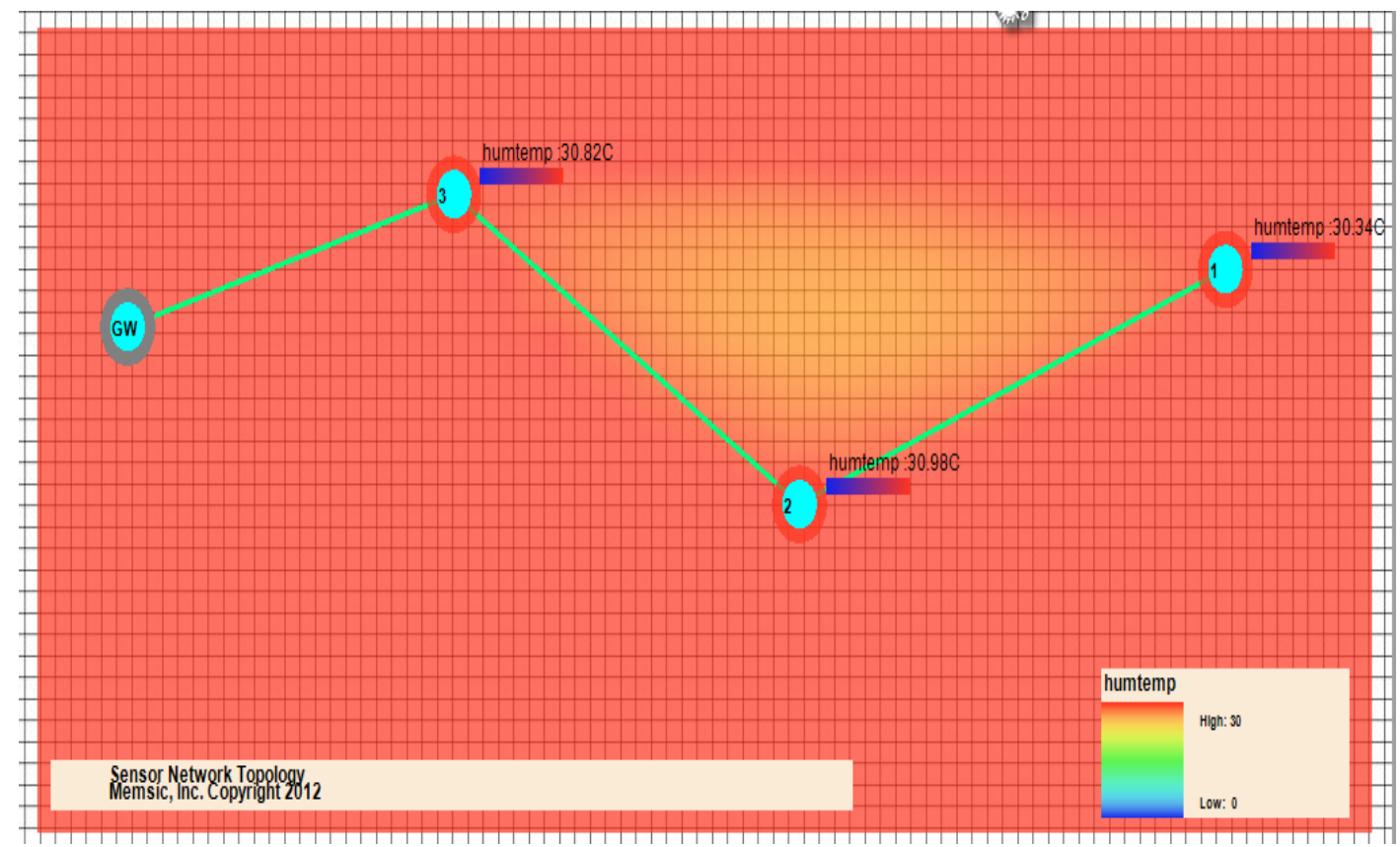

Fig. 4.Multi Hop Topology of the network

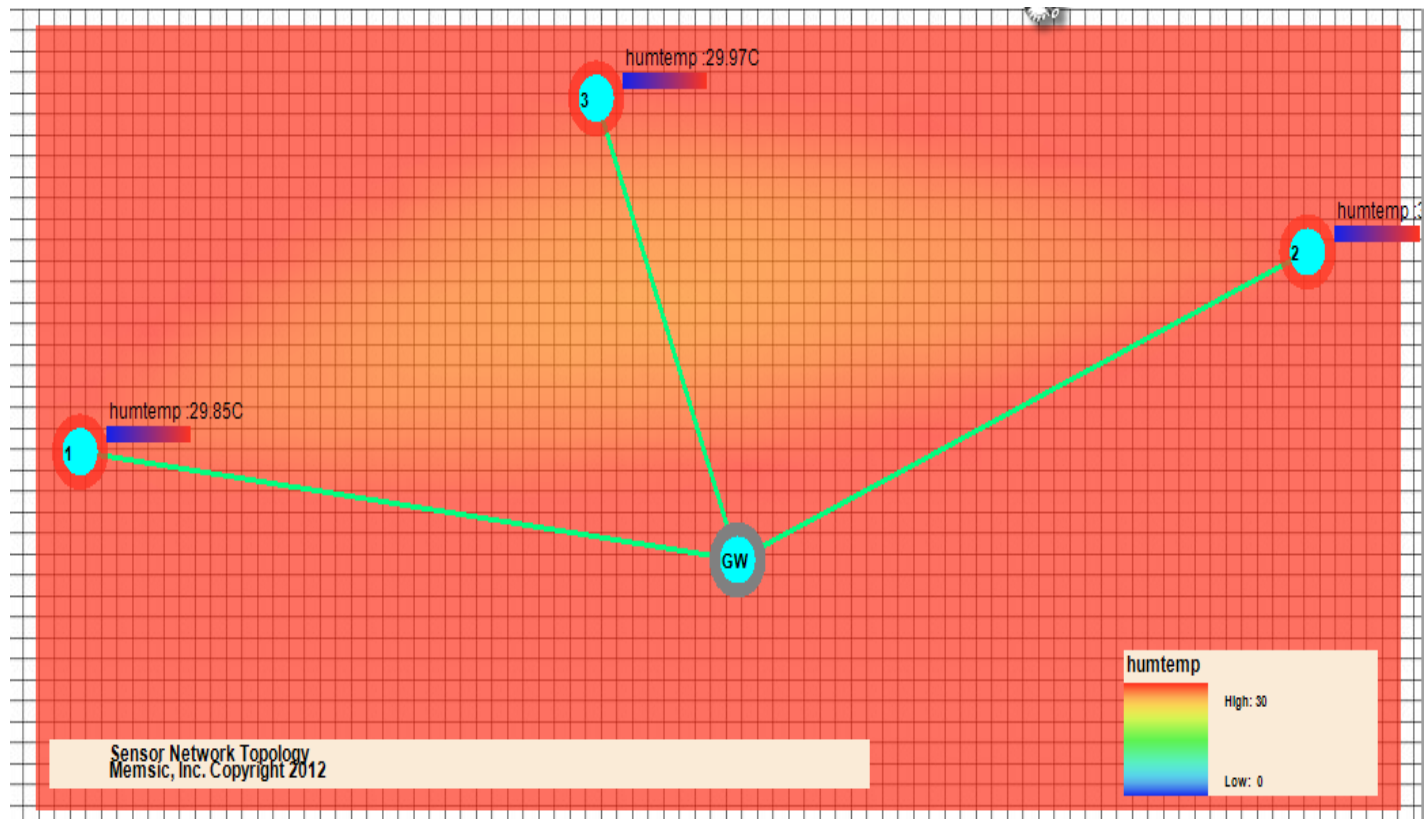

Fig. 5.Star Topology of the network

The sensor nodes are kept in different places in a home automation context. Now the information from three different nodes is collected .The below Figure shows the network topology in both multi hop and star topologies. The multihop is established when the nodes are far away from the central node. We can get different values from temperature and humidity from different environments.

\section{RESULTS}

The temperature values are indicated with color coding. The color red indicates values of high temperature and when there is a low value green color is represented. In general, the node1 can be placed near a heat source and program to manage an alarm if any data detected reach maximum value or exceed a threshold value. Figure 7 shows data that was retrieved from the whole network and saved to a PostgreSQL database. 


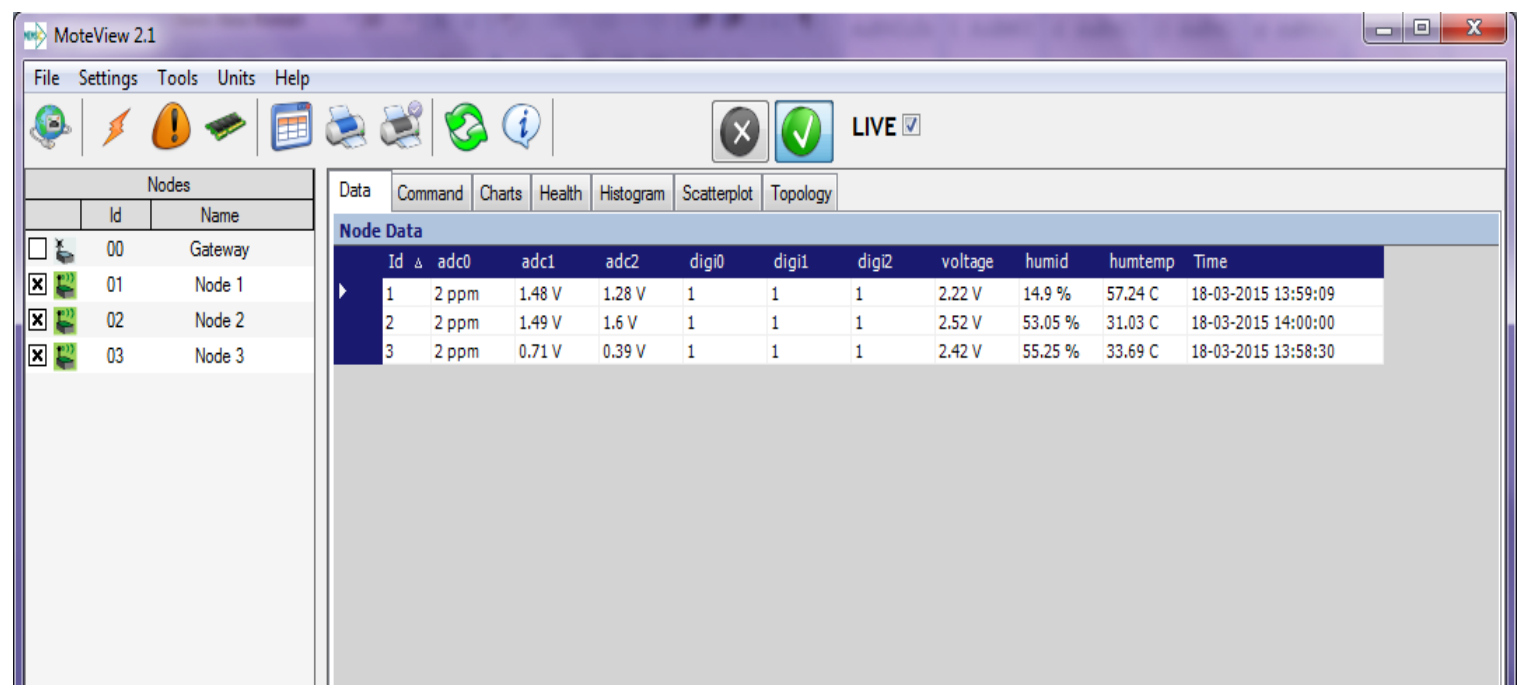

Fig. 6. Data table in Moteview

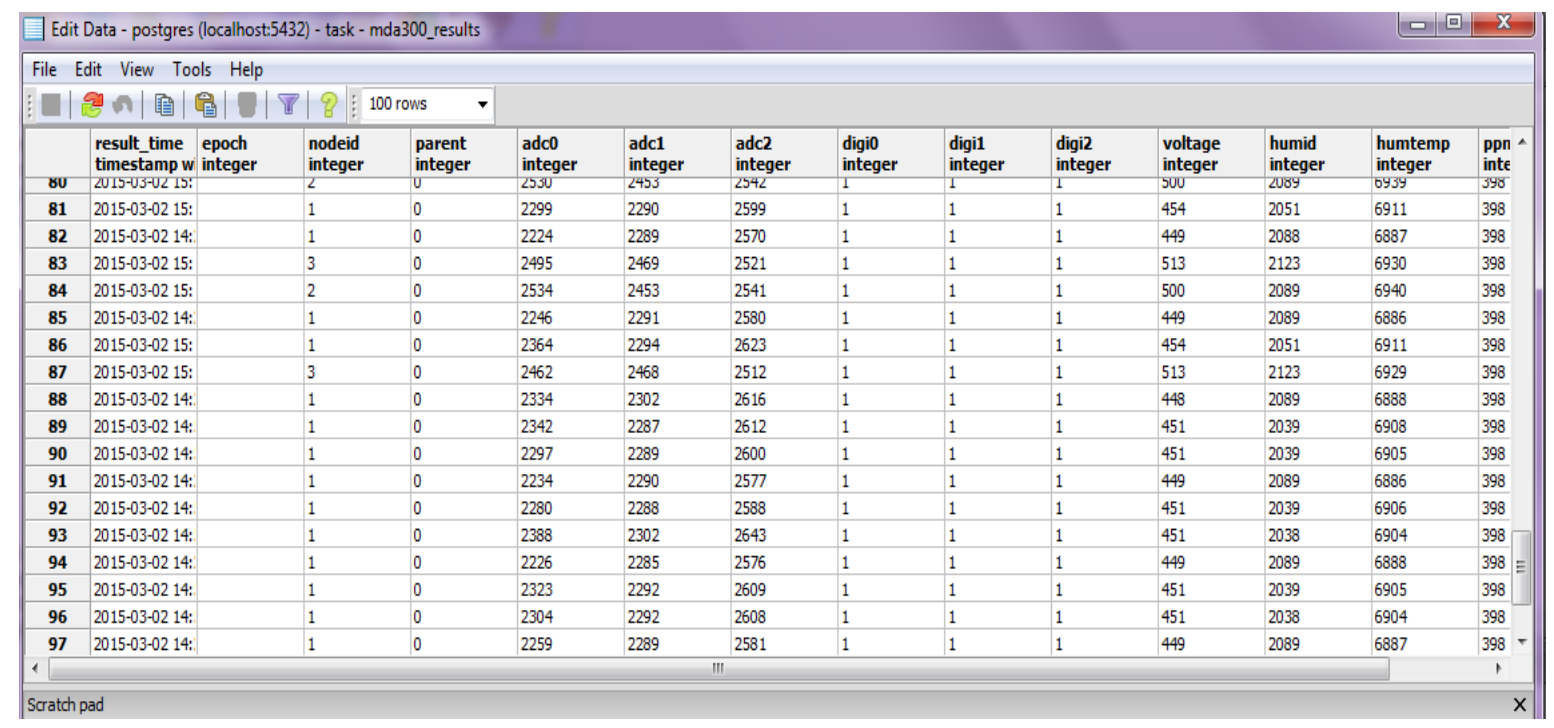

Fig. 7. Data Base Table in PostGRE SQL

Information saved for each node are result_time, nodeid, voltage, humid, humtemp. The Moteview also measures the parameters in communication like signal power, packet loss percentage and packets forwarded percentage. The charts and histogram provide the percentage of fixed values.

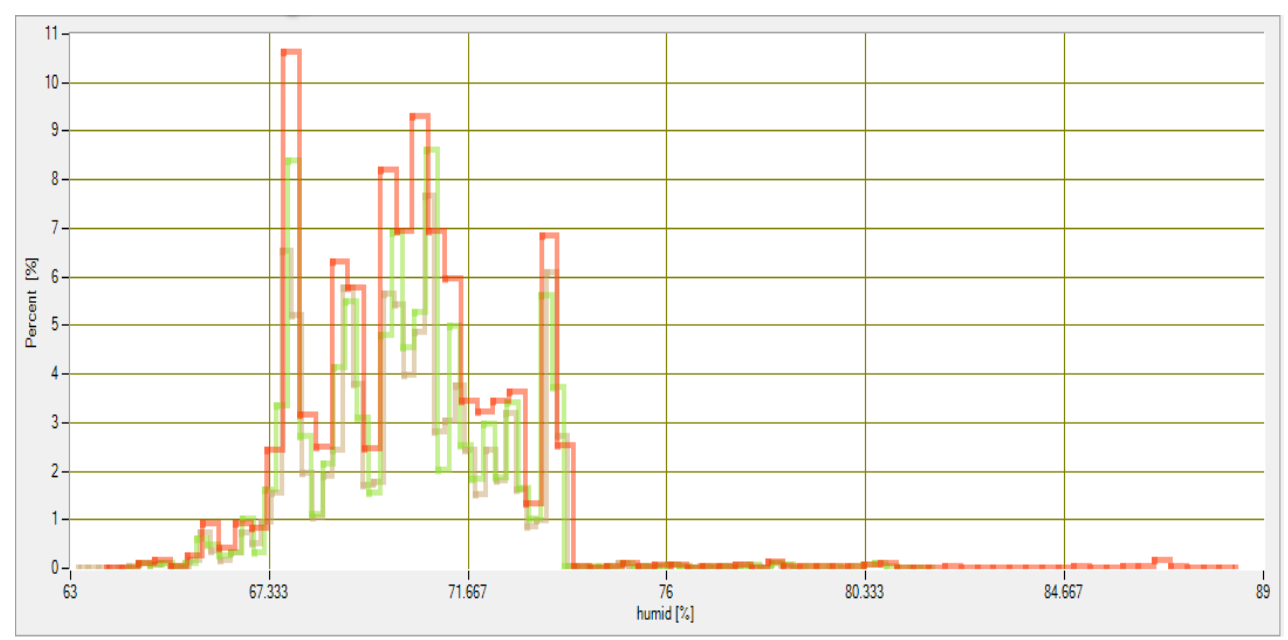

Fig. 8. Histogram value 


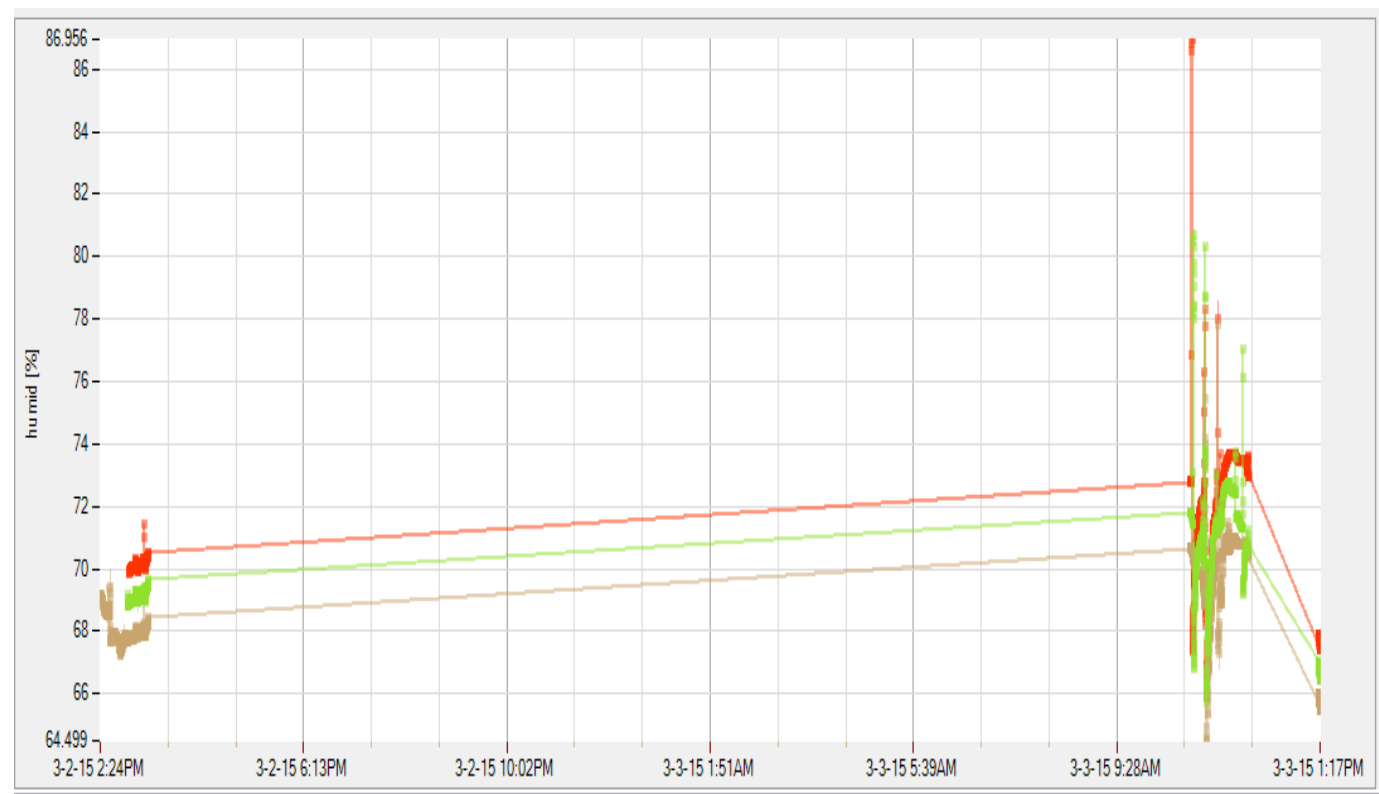

Fig. 9. Charts Based on Results

\section{CONCLUSION AND FUTURE WORK}

Our paper describes the architecture and design of WSN's for indoor and environment applications using the moteworks software and with the help of moteview data can be seen. The advantage of this design is simple to use and we can create a WSN which can work perfectly and monitor the area and received information can be stored into the database. Furthermore, we can work to test network performances in industrial applications. We can also connect external sensors to the DAC and further it can be used in real-time environments.

\section{REFERENCES}

[1] Crossbow, "MoteConfig Users Manual", Revision A, November 2006, PN: 7430-0112-01

[2] Crossbow, "MPR-MIB Users Manual", Revision A, June 2007, PN: 7430-0021-08

[3] Crossbow, "MTS/MDA Sensor Board Users Manual",

[4] Revision A, June 2007, PN: 7430-0020-05

[5] Crossbow "MoteWorks Getting Started Guide", Revision D, March 2007, PN: 7430-0102-01

[6] ZigBeeAlliance Home, http://www.zigbeealliance.org (last access 5 March 2012)

[7] WikiStart-Nano-RK, http://www.nanork.org/projects/ nanork/wiki (last access 16 February 2012)

[8] Bluetooth Specification Version 3.0 + HS; Bluetooth SIG 2009

[9] IEC 62591 Ed. 1.0: Industrial communication networks - Wireless communication network and communication profiles - WirelessHART (FDIS).

[10] IEEE Std 802.11-2007 for Information technology Telecommunications and information exchange between systems - Local and metropolitan area networks - Specific requirements - Part 11: Wireless LAN Medium Access Control (MAC) and Physical
Layer (PHY) Specifications, C1-1184, June 2007.

[11] Q. Zhang et.al, "Application of WSN in precision forestry",

[12] Electronic Measurement \& Instruments (ICEMI), August 2011

[13] MEMSIC: Wireless Sensor Networks, eKo, Imote2, MICAz, TelosB, and IRIS Wireless Development Kits, http://www.memsic.com/ (last access 1 March 2012)

[14] A.A. Abed et.al, "Building an HMI and demo application of WSN-based industrial control systems", Energy, Power and control (EPC-IQ), May 2011

[15] The Contiki OS, http://www.contiki-os.org/ (last access 15 February 2012)

[16] TinyOS Home Page, http://www.tinyos.net/ (last access 4 March 2012)

\section{BIOGRAPHIES}

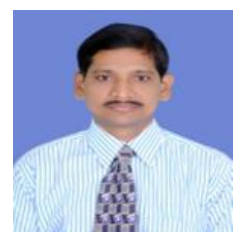

K.Raghava Rao, Professor in CSE, working in the dept. of ECM. He is having 15 years of experience in teaching for $\mathrm{UG}$ and $\mathrm{PG}$ engineering students and 3 years of Software Development experience in Singapore. He received B.E(CSE) from MG state University, M.Tech (CSE) from RVP University, Udaipur and PhD (CSE) from Mahatma Gandhi University (Kasi Vidyapeeth), Varanasi, in the years 1995, 2005 and 2009 respectively. He published several papers in national \& international conferences and journals. He published 3 text books. Currently he is carrying a DST funded project in the area of Wireless sensor networks-sensor web enablement. His research interests are Wireless sensor networks, Embedded Sensor networks and Sensor Web Enablement. He received a best paper award from DST Secretary and Best Teacher award from KL University $\mathrm{He}$ is also member of several technical organizations. 
G.G.Raja sekhar, studying B. Tech final year in the dept. of ECM in K L University. His area of interests is

Embedded systems and database management system. He has done projects on client/server system technology based on TCP/IP protocols and Database

Management System (DBMS). He also attended the workshop on Sensor Networks and also done internship for one month on Basics of Embedded systems.

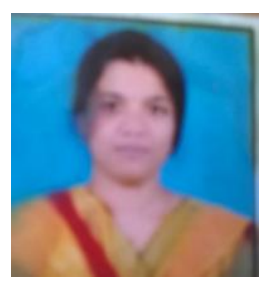

J.Varsha, studying B. Tech final year in the dept. of ECM in K L University. Her area of interests is Embedded systems and database management system. She has done projects on client/server system technology based on TCP/IP protocols and Database Management System (DBMS). She also attended the workshop on Network security and also done internship for two months on Computer Networks. 